\title{
Foot Care Knowledge and Practice among Diabetic Patients Attending General Outpatient Clinic in Tribhuvan University Teaching Hospital
}

\author{
Tirtha Man Shrestha1 ${ }^{*}$, Ramesh P. Aacharya1, Rabina Shrestha², Madhav KC ${ }^{3}$ \\ ${ }^{1}$ Department of Emergency and General Practice, Tribhuvan University Teaching Hospital, Kathmandu , Nepal \\ ${ }^{2}$ Tripler Army Medical Center, Honolulu, HI, USA \\ ${ }^{3}$ Louisiana State University Health Sciences Center, New Orleans, LA, USA \\ Email: *tirtha_48@yahoo.com.au
}

How to cite this paper: Shrestha, T.M., Aacharya, R.P., Shrestha, R. and KC, M. (2017) Foot Care Knowledge and Practice among Diabetic Patients Attending General Outpatient Clinic in Tribhuvan University Teaching Hospital. Open Journal of Endocrine and Metabolic Diseases, 7, 163-171. https://doi.org/10.4236/ojemd.2017.78015

Received: July 23, 2017

Accepted: August 22, 2017

Published: August 25, 2017

Copyright $\odot 2017$ by authors and Scientific Research Publishing Inc. This work is licensed under the Creative Commons Attribution-NonCommercial International License (CC BY-NC 4.0). http://creativecommons.org/licenses/by-nc/4.0/

\begin{abstract}
Background: Diabetes Mellitus results in several serious complications and among them foot problem is one of the commonly ignored complications. This study is an attempt to find out the level of patient's awareness and practice in Nepalese context. Objective: The aim of this study is to determine the knowledge and practice of foot care among patients with diabetes in Nepal. Methods: This is a six-month long cross-sectional study done in the General Practice Clinic of Tribhuvan University Teaching Hospital. Diabetic patients were given structure questionnaires on knowledge, foot care practices, and demographic information. Results: Illiteracy was found be significantly associated with poor knowledge and practice of foot care. Those who have no education are more likely to have poor knowledge and foot care practice. The odds of having poor knowledge is $6.414(3.075,13.379)$ in those who have no education/primary education compared to those who have education and the odds of poor foot care practice is $4.5180(2.077,8.411)$ in those who have no education/primary education compared to those who have education with significance of $<0.001$. Conclusion: This study has highlighted the need of appropriate education intervention for the patients with diabetes who have no education background to prevent from foot complications. Health education materials such as graphics, photos are needed to make them comprehensible for the illiterate patients.
\end{abstract}

\section{Keywords}

Diabetes, Complications, Illiteracy, Knowledge, Practice, and Intervention 


\section{Introduction}

Diabetes Mellitus (DM) is a metabolic disorder that is characterized by chronic hyperglycemia; it is a common and potentially disabling chronic disease [1] [2]. This non-communicable disease is a global public health threat that has increased dramatically over the past two decades [3] [4]. The global prevalence of diabetes has nearly doubled since 1980 , rising $4.7 \%$ to $8.5 \%$ in the adult population [5].

Diabetic foot ulcer (DFU) is a full thickness wound penetrating through the dermis located below the ankle in a diabetes which can become infected it is untreated [6]. Foot ulcer is one the major complications diabetes patients have which can be prevented with good care of feet. Triad of neuropathy, angiopathy, and trauma will make the patients of Diabetes Mellitus vulnerable to diabetic foot ulcer [7]. People with diabetes have a $25 \%$ lifetime risk of developing foot ulcers, which can develop into chronic non-healing ulcers [7] [8].

Diabetes complications, such as DFU and amputation, have a negative impact on quality of life (QOL) [9] [10]. Most lower limb amputations in patients with diabetes are preceded by a foot ulcer, whose risk factors apart from peripheral vascular disease (PVD) and peripheral neuropathy (PN), are barefoot walking, inappropriate footwear, poor foot hygiene and delay in seeking medical attention [11]. Study conducted in Nepal found 58\% prevalence of diabetic sensory peripheral neuropathy [12]. Foot complications increase the risk for amputation in diabetes by 12.3 folds as compared to the normal population [13].

Study has shown that majorities of people with diabetes do not receive guideline-recommended foot care, including regular foot examinations [14]. All patients, if given proper guidance and education regarding foot care, should be able to make significant improvements in their foot care [15]. It has been estimated that up to $50 \%$ of the major amputations in diabetic patients can be prevented with effective education [6].

There are limited studies done in Nepal on foot care among diabetic patients. Therefore, this study will help to assess the knowledge and practice of foot care.

\section{Materials and Methods}

1) Study design and population: This is an observational cross-sectional study conducted at General Practice Clinic of Tribhuvan University Teaching Hospital, Kathmandu, Nepal. Participants of the study are those who are diagnosed with diabetes and being treated in the Hospital.

2) Sample size: To avoid the selection bias all diabetic patients attending GP clinic during the study period were enrolled in the study. A total of 196 diabetic patients were included in this study.

3) Tools: Structured research questionnaire developed by diabetes research group, University of Newcastle was translated into Nepali language. It included 11 questions related to knowledge and 11 questions of practices of foot care. The Nepali version was pilot tested in 15 diabetic patients for the validity and relia- 
bility of structured questionnaires. Minimal revisions had to be done after the pilot study.

4) Ethical considerations: The study was approved by the Institutional Review Board of the Institute of Medicine. Written informed consent was obtained from the participants and then, the questionnaire was given to the participants. Illiterate patients received assistance from the clinic nurse to answer the questionnaire.

5) Outcome variable: Poor knowledge and poor foot care practice were two outcome variables. There were 11 questions on knowledge of diabetes, based on that score for knowledge of foot care was created ranging from 0 to 11 , similarly score for foot care practices were created based on their foot care practice ranging from 2 to 10 . Knowledge score and practice score were classified into two categories good and poor. Score $\geq 70 \%$ ( 8 - 11) was considered as good whereas score $<70 \%(1-7)$ was considered as poor.

6) Exposure variable: The main predictor of the interest was poor education (Illiteracy). The two categories of the education were no/primary education (Illiterate) and secondary/higher (literate).

7) Covariates: Some of the covariates of the study were gender and age. Age was categorized into two groups i.e. the age greater or equal to 50 years and the age less than 50 years.

8) Statistical analysis: Data were reviewed and verified before performing any statistics to prevent human errors. SAS 9.4 was used to perform all analysis of the study. P-value less than 0.05 was considered as significance level. Descriptive statistics was performed to get frequency and percentage distribution of demographic factors. Chi-square test was used to compare the differences between two score groups according to knowledge and practice. Furthermore, logistics regression was performed to determine the association between education with knowledge and practice.

\section{Results}

Demographic characteristics are depicted in Table 1. The total number of participants was 196 , out of which $53.06 \%$ were aged $\geq 50$ years. The age range of age of the participants was 30 to 85 . Out of total study population, 108 (55.10\%) were male and $88(44.90 \%)$ were female. More than half of the study population $(63.78 \%)$ were not educated at all or had primary education whereas only $36.32 \%$ had secondary or higher education. Twenty six percent of them had hypertension as well. About $17 \%$ of participants have both hypertension and dyslipidemia along with diabetes.

Responses on foot care knowledge are tabulated in Table 2. The mean knowledge score was $7.03(6.6057 \pm 7.4555)$. About one fourth (24.49\%) did not know that they need to look after foot since they might injure due to inability to sense, and one third of the patients (33.67\%) of the patients did not know they might get ulcers if foot is not cared properly. About two-third (64.29\%) of the diabetes 
Table 1. Characteristics of patients in the study $(n=196)$.

\begin{tabular}{lc}
\hline Characteristics & Frequency $(\mathrm{N})$ \\
\hline Age (mean \pm SD) & $52.9 \pm 12.10$ years \\
$<50$ years & $92(46.94 \%)$ \\
$\geq 50$ years & $104(53.06 \%)$ \\
Sex & \\
Male & $108(55.10 \%)$ \\
Female & $88(44.90 \%)$ \\
Education & \\
None/primary & $125(63.78 \%)$ \\
Secondary or higher & $71(36.22 \%)$ \\
Comorbidity & \\
Hypertension & $52(26.53 \%)$ \\
Dyslipidemia & $10(5.10 \%)$ \\
Chronic Obstructive Pulmonary Disease (COPD) & $4(2.04 \%)$ \\
Hypothyroidism & $3(1.53 \%)$ \\
None & $69(35.20 \%)$ \\
\hline
\end{tabular}

Table 2. Response on foot care knowledge questionnaire $(n=196)$.

\begin{tabular}{lcc}
\hline $\begin{array}{l}\text { Questions related to knowledge of foot care among } \\
\text { diabetic patient }\end{array}$ & $\begin{array}{c}\text { Know/Correct } \\
\text { (\%) }\end{array}$ & $\begin{array}{c}\text { Don't know/Incorrect } \\
\text { (\%) }\end{array}$ \\
\hline $\begin{array}{l}\text { DM patients should take medication regularly because } \\
\text { they liable to get DM complication }\end{array}$ & 84.18 & 15.82 \\
$\begin{array}{l}\text { DM patients should look after their feet because they may } \\
\text { not feel a minor injury to their feet }\end{array}$ & 75.51 & 24.49 \\
$\begin{array}{l}\text { DM patients should look after their feet because wounds } \\
\text { and infection may not heal quickly }\end{array}$ & 81.12 & 18.88 \\
$\begin{array}{l}\text { DM patients should look after their feet because they may } \\
\text { get a foot ulcer }\end{array}$ & 66.33 & 33.67 \\
$\begin{array}{l}\text { DM patients should not smoke because smoking causes } \\
\text { poor circulation affecting the feet }\end{array}$ & 69.90 & 18.88 \\
$\begin{array}{l}\text { How often do you think you should inspect your feet } \\
\text { If you found redness/bleeding between your toes what is } \\
\text { the first thing you do }\end{array}$ & 81.12 & 64.29 \\
$\begin{array}{l}\text { Even if you have never had a corn/ hard skin lesion, what } \\
\text { would you do if you had one }\end{array}$ & 26.53 & 73.71 \\
$\begin{array}{l}\text { How often do you think your feet should be Washed } \\
\text { What temperature of water do you think you should wash } \\
\text { your feet in }\end{array}$ & 76.53 & 26.14 \\
$\begin{array}{l}\text { How often do you think you should inspect the inside of } \\
\text { your footwear for objects or torn lining }\end{array}$ & 73.98 & 67.86 \\
\hline
\end{tabular}


patients did not know what is the first thing to do when they find redness/ bleeding between their toes? Likewise, $67.86 \%$ were unaware of water temperature that they should have to clean their feet. Most of the patients (73.47\%) had no knowledge on what should be done if they develop corn/hard skin lesion.

Responses on foot care practices are tabulated in Table 3. The mean practice score was $5.31(4.9990 \pm 5.6235)$. Half of the diabetes patients $(50.51 \%)$ did not receive advice on footwear and $61.22 \%$ did not measure their feet measured when they bought footwear last. Some of the other poor foot care practices were; $68.88 \%$ of the respondents do not wash their feet with warm water, $30.61 \%$ walk barefoot regularly, $26.53 \%$ do not inspect their footwear before wearing, and $25.51 \%$ do not wash their feet regularly.

Knowledge and practice scores are significantly correlated ( $\mathrm{p}$-value $<0.0001$ ) with correlation coefficient of 0.85541 . Since knowledge and practice scores are positively correlated it suggests that if knowledge score increases practice score increases and vice versa. From the Pearson correlation coefficient, we can conclude that knowledge and practice score have strong relationship.

Chi square test was performed to compare the proportion of variables of interest (education, age, and gender) by knowledge and practice score. Both exposure and outcome variables were categorized into 2 groups. For both knowledge and practice scores, $\geq 70 \%$ score set as good and $<70 \%$ as poor groups. There was significant difference between illiterate and literate participants by knowledge score as well as practice score. Out of 196 diabetic patients who have no formal education are more likely to have poor knowledge compared to those who have secondary/higher education $(63.20 \%$ vs. $22.54 \%$, p-value $<0.0001)$. Also, patients with no/primary education are more likely to have poor foot care practice compared to those who have secondary/higher education (57.60\% vs. $23.94 \%$,

Table 3. Response on foot care practice questionnaire $(n=196)$.

\begin{tabular}{lcc}
\hline Questions related to practice of foot care among diabetic patient & Yes (\%) & No (\%) \\
\hline Do you Inspect feet regularly & 80.61 & 19.39 \\
Do you wash feet regularly & 74.49 & 25.51 \\
Do you wash feet with warm water & 31.12 & 68.88 \\
Do you trim toe nails straight across & 82.65 & 17.35 \\
Do you measure your feet size when last you bought Footwear & 38.78 & 61.22 \\
Did you receive advice when you last bought footwear & 49.49 & 50.51 \\
Did you ever inspect inside of footwear & 73.47 & 26.53 \\
Do you regularly walk barefoot & 30.61 & 69.39 \\
Do you clean nails with sharp instrument & 13.78 & 86.22 \\
Do you add irritants to water before feet cleaning & 3.06 & 96.94 \\
Do you wear elasticated socks & 53.61 & 46.39 \\
\hline
\end{tabular}


p-value $<0.0001)$. Gender was found to be significant on both knowledge and practice score. Females tend to have poor knowledge $(60.23 \%$ vs. $38.89 \%$, p-value $<0.0029)$ and practice $(55.68 \%$ vs. $37.04 \%$, p-value $<0.0093)$ compared to males. However, age did not have any significant effect on knowledge and practice of study population. The comparison analysis is shown in Table 4.

The multivariate logistic regression was performed to find the association between demographic factors and knowledge and practice score (Table 5). The main predictor of poor practice of foot care among diabetic patient was found to be educational level.

Those who were not educated had odds of $6.414(3.075,13.379)$ of having poor knowledge compared to those who have secondary/higher education. The odds of poor foot care practice in patients with no/primary education was 4.180 $(2.077,8.411)$ compared to those with secondary/higher education. Therefore, diabetic patients with no education are more likely to have poor knowledge and poor practice of foot care. No significant association was seen between age and gender with knowledge and practice score.

\section{Discussion}

This study shows about half of the study patients do not have good knowledge and practice on foot care which indicates they are at higher risk of foot complications. This study also illustrates the lack of education is significantly associated with poor foot care knowledge and practice. The finding of this study supports the study done in Nepal by Agrawaal where educational status was significantly associated with awareness of foot care [16].

Diabetic patients lack knowledge on what to do when there is redness/bleeding between toes, temperature to wash feet, and what to do if there is

Table 4. Differences in knowledge and foot care practice according to level of education $(\mathrm{n}=196)$.

\begin{tabular}{|c|c|c|c|c|}
\hline Characteristics & Poor foot care knowledge & $\mathrm{p}$-value & Poor foot care practice & p-value \\
\hline Illiterate & $63.20 \%$ & \multirow[b]{2}{*}{$<0.0001$} & $57.60 \%$ & \multirow[b]{2}{*}{$<0.0001$} \\
\hline Literate & $22.54 \%$ & & $23.94 \%$ & \\
\hline Age $\geq 50$ & $47.12 \%$ & \multirow[b]{2}{*}{0.6867} & $46.15 \%$ & \multirow[b]{2}{*}{0.8236} \\
\hline Age $<50$ & $50 \%$ & & $44.57 \%$ & \\
\hline Female & $60.23 \%$ & \multirow[b]{2}{*}{0.0029} & $55.68 \%$ & \multirow[b]{2}{*}{0.0093} \\
\hline Male & $38.89 \%$ & & $37.04 \%$ & \\
\hline
\end{tabular}

Table 5. Association between demographic factors with knowledge and practice of foot care $(\mathrm{n}=196)$.

\begin{tabular}{ccccc}
\hline Predictors & Poor knowledge (Adjusted OR) & p-value & Poor practice (Adjusted OR) & p-value \\
\hline Illiterate & $6.414(3.075,13.379)$ & $<0.0001$ & $4.180(2.077,8.411)$ & $<0.0001$ \\
Age $\geq 50$ & $0.514(0.264,1.000)$ & 0.0500 & $0.731(0.390,1.368)$ & 0.3268 \\
Female & $1.636(0.867,3.088)$ & 0.1290 & $1.542(0.832,2.857)$ & 0.1690 \\
\hline
\end{tabular}


corn/hard skin lesion in the skin. Having no education is associated with poor knowledge i.e. $63.20 \%$ of no education (illiterate) patients have poor knowledge. Out of 196 patients, $23.98 \%$ have poor knowledge score $(<50 \%)$. This finding is consistent with the study done in Tanzania [15]. The higher the education more knowledgeable patients seem to be; this may be because educated patients are able to read and easily grasps the valuable information about foot care. Also, they might be able to understand how important it is to prevent foot complications and to take care of their own health compared to those who are not educated.

In this study, $20.92 \%$ of patients have poor practice ( $<50 \%$ score), which resembles with Gholap et al. study where $20 \%$ had poor practice [17]. In contradiction, $51 \%$ of patients in Sutariya et al. study and $49.4 \%$ of patients in Desalu et al. study had poor practice [17] [18]. Some of the risky foot care practices patients reported in this study are not washing feet with warm water $(68.88 \%)$, not measuring the size of feet while buying foot wear $(61.22 \%)$, wearing elasticated socks (46.39\%), and walking barefoot (30.61\%).

We also found that male patients tend to have good knowledge and practice of foot care compared to female patients. This result contradicts with the result of Pollock et al. study where females had significant higher knowledge score than male [19]. This indicates females are more susceptible to have foot complications compared to males. However, odds ratio was not found to be significant even there was differences in proportion between females and males by poor practice score.

Communication between health care providers and patients can also help to improve the patients. Patients who have poor foot care practices may have never received any information regarding taking care of feet. Insufficient or no advice from the doctors might have affected patients' ability to take care of their feet. Thorough examination and detail instructions for foot care from doctors and nurses can influence patients' self-examination along with proper care of feet. Study conducted by Pollock et al has shown patients who received advice on foot self-examination showed significantly greater practice of examining the feet [19].

This study emphasizes the importance of education to improve overall health. Patients with DFU should be educated about risk factors and the importance of foot care, including the need for self-inspection, monitoring foot temperature, appropriate daily foot hygiene, use of proper footwear, and blood sugar control [20]. Moreover, our study has highlighted the fact that education can significantly increase the level of knowledge and good practice on foot care. Our study results supported other studies done in different countries. The findings can be used to develop education program for illiterate diabetic patients to prevent the diabetes related foot complications.

\section{Limitations of the Study}

1) The study was conducted in university hospital, it may not represent the 
community situation which is important in terms of knowledge and practice.

2) This is a single center study. Large scale multicenter study is needed to represent the national situation.

3) Age might be confounding factor. Young age group might be more educated and aware of disease condition.

\section{Conclusion}

Having no education/primary education was significantly associated with patient's foot care knowledge and practice. To prevent foot complications and amputation in diabetic patients, it is important to have foot care knowledge. Both knowledge and practice are dependent on patients' education. Since patients without education tend to have poor knowledge and practice, easily comprehensible health education and promotion program on foot care should be included in all clinics taking care of diabetic patients.

\section{Acknowledgements}

I am grateful to Dr. Jachin Velavan (The coordinator Department of Distance Education) and Dr. Nihal Thomas (Head of Department of Endocrinology) for allowing and encouraging me to undergo this work.

\section{References}

[1] Eastman, R.C., Javitt, J.C., Herman, W.H., Dasbach, E.J., Copley-Merriman, C., Maier, W., et al. (1997) Model of Complications of NIDDM. II. Analysis of the Health Benefits and Cost-Effectiveness of Treating NIDDM with the Goal of Normoglycemia. Diabetes Care, 20, 735-744. https://doi.org/10.2337/diacare.20.5.735

[2] Wild, S., Roglic, G., Green, A., Sicree, R. and King, H. (2004) Global Prevalence of Diabetes: Estimates for the Year 2000 and Projections for 2030. Diabetes Care, 27, 1047-1053. https://doi.org/10.2337/diacare.27.5.1047

[3] Ramachandran, A., Snehalatha, C., Shetty, A.S. and Nanditha, A. (2012) Trends in Prevalence of Diabetes in Asian Countries. World Journal of Diabetes, 3, 110-117. https://doi.org/10.4239/wjd.v3.i6.110

[4] Shahbazian, H., Yazdanpanah, L. and Latifi, S.M. (2013) Risk Assessment of Patients with Diabetes for Foot Ulcers According to Risk Classification Consensus of International Working Group on Diabetic Foot (IWGDF). Pakistan Journal of Medical Sciences, 29, 730-734. https://doi.org/10.12669/pjms.293.3473

[5] World Health Organization (2016) Global Report on Diabetes. Geneva.

[6] Seid, A. and Tsige, Y. (2015) Knowledge, Practice, and Barriers of Foot Care among Diabetic Patients Attending Felege Hiwot Referral Hospital, Bahir Dar, Northwest Ethiopia. Advances in Nursing, 2015, Article ID: 934623. https://doi.org/10.1155/2015/934623

[7] Kasilambros, N., Dounis, E., Makrilakis, K., Tentolouris, N. and Tsapogas, P. (2010) Atlas of the Diabetic Foot. 2nd Edition, Wiley-Blackwell, Hoboken, NJ. https://doi.org/10.1002/9781444317589

[8] Lavery, L.A., Armstrong, D.G., Wunderlich, R.P., Tredwell, J. and Boulton, A.J. (2003) Diabetic Foot Syndrome: Evaluating the Prevalence and Incidence of Foot 
Pathology in Mexican Americans and Non-Hispanic Whites from a Diabetes Disease Management Cohort. Diabetes Care, 26, 1435-1438. https://doi.org/10.2337/diacare.26.5.1435

[9] Ragnarson-Tennvall, G. and Apelqvist, J. (2000) Health-Related Quality of Life in Patients with Diabetes Mellitus and Foot Ulcers. Journal of Diabetes and Its Complications, 14, 235-241. https://doi.org/10.1016/S1056-8727(00)00133-1

[10] Ribu, L., Hanestad, B.R., Moum, T., Birkeland, K. and Rustoen, T. (2007) A Comparison of the Health-Related Quality of Life in Patients with Diabetic Foot Ulcers, with a Diabetes Group and a Nondiabetes Group from the General Population. Quality of Life Research, 16, 179-189. https://doi.org/10.1007/s11136-006-0031-y

[11] Abbas, Z.G. and Archibald, L.K. (2007) Challenges for Management of the Diabetic Foot in Africa: Doing More with Less. International Wound Journal, 4, 305-313. https://doi.org/10.1111/j.1742-481X.2007.00376.x

[12] Karki, D.B., Yadava, S.K., Pant, S., Thusa, N., Dangol, E. and Ghimire, S. (2016) Prevalence of Sensory Neuropathy in Type 2 Diabetes Mellitus and Its Correlation with Duration of Disease. Kathmandu University Medical Journal, 54, 120-124.

[13] Morris, A.D., McAlpine, R., Steinke, D., Boyle, D.I., Ebrahim, A.R., Vasudev, N., et al. (1998) Diabetes and Lower-Limb Amputations in the Community. A Retrospective Cohort Study. DARTS/MEMO Collaboration. Diabetes Audit and Research in Tayside Scotland/Medicines Monitoring Unit. Diabetes Care, 21, 738-743. https://doi.org/10.2337/diacare.21.5.738

[14] Barshes, N.R., Sigireddi, M., Wrobel, J.S., Mahankali, A., Robbins, J.M., Kougias, P., et al. (2013) The System of Care for the Diabetic Foot: Objectives, Outcomes, and Opportunities. Diabetic Foot \& Ankle, 4, Article: 21847.

https://doi.org/10.3402/dfa.v4i0.21847

[15] Chiwanga, F.C. and Njelekela, M.A. (2015) Diabetic Foot: Prevalence, Knowledge, and Foot Self-Care Practices among Diabetic Patients in Dar es Salaam, Tanzania-A Cross-Sectional Study. Journal of Foot and Ankle Research, 8, 20. https://doi.org/10.1186/s13047-015-0080-y

[16] Agrawaal, K.K. (2015) Patients' Awareness about the Complications of Diabetes Mellitus and Its Correlation with the Glycemic Status. Journal of Nepal Medical Association, 53, 284-287.

[17] Hasnain, S. and Sheikh, N. (2009) Knowledge and Practices Regarding Foot Care in Diabetic Patients Visiting Diabetic Clinic in Jinnah Hospital, Lahore. The Journal of Pakistan Medical Association, 59, 4.

[18] Muhammad-Lutfi, A.R., Zaraihah, M.R. and Anuar-Ramdhan, I.M. (2014) Knowledge and Practice of Diabetic Foot Care in an In-Patient Setting at a Tertiary Medical Center. Malaysian Orthopaedic Journal, 8, 22-26. https://doi.org/10.5704/MOJ.1411.005

[19] Pollock, R.D., Unwin, N.C. and Connolly, V. (2004) Knowledge and Practice of Foot Care in People with Diabetes. Diabetes Research and Clinical Practice, 64, 117-122. https://doi.org/10.1016/j.diabres.2003.10.014

[20] Tseng, K.H. (2006) Standards of Medical Care in Diabetes-2006: Response to the American Diabetes Association. Diabetes Care, 29, 2563-2564.

https://doi.org/10.2337/dc06-0805 